\title{
Constituintes voláteis de Mentha pulegium L. e Plectranthus amboinicus (Lour.) Spreng.
}

\author{
OLIVEIRA, R.A. ${ }^{1 *}$; SÁ, I.C.G. ${ }^{1}$; DUARTE, L.P. ${ }^{2}$; OLIVEIRA, F.F. ${ }^{1}$ \\ ${ }^{1}$ Departamento de Ciências Exatas e Tecnológicas, Universidade Estadual de Santa Cruz, Rodovia Ilhéus-Itabuna, \\ Km 16, Salobrinho, CEP: 45656-900, Ilhéus-Brasil *rosilene@uesc.br ${ }^{2}$ Departamento de Química, Universidade \\ Federal de Minas Gerais, Avenida Carlos Luz, 6627, Pampulha, CEP:31270-901, Belo Horizonte-Brasil
}

\begin{abstract}
RESUMO: Nesse trabalho foi investigado a composição química de óleos essenciais de duas plantas medicinais cultivadas em Ilhéus, Brasil, Plectranthus amboinicus (Lour.) Spreng. (hortelãgrosso) e Mentha pulegium L. (poejo). Os óleos essenciais foram extraídos das folhas frescas por hidrodestilação e foram analisados por CG/FID e CG-EM, na primavera e no inverno. $P$. amboinicus forneceu, tanto na primavera como no inverno, $0,10 \%$ de óleo sendo timol o componente majoritário. M. pulegium forneceu na primavera $0,20 \%$ de óleo e no inverno $0,09 \%$. Os componentes majoritários dos óleos foram pulegona e trans-cariofileno; borneol, mentol e piperitona foram identificados em menores quantidades. No inverno foram observados maiores quantidades de mentol e isomentol. Acetatos de neoisomentila, de mentila e de isometila foram observados somente no inverno. Esse trabalho contribui para o conhecimento das espécies vegetais cultivadas na região do sul da Bahia.
\end{abstract}

Palavras-chave: Lamiaceae, llhéus, Bahia, primavera, inverno

\begin{abstract}
Volatile constituents of Mentha pulegium L. and Plectranthus amboinicus (Lour.) Spreng. This work investigated the chemical composition of the essential oils of two medicinal plants grown in Ilhéus Municipality, Bahia State, Brazil: Plectranthus amboinicus (Lour.) Spreng (Mexican mint) and Mentha pulegium L. (pennyroyal). The essential oils were extracted from fresh leaves by hydrodistillation and analyzed by GC-FID and GC-MS, in the spring and in the winter. In both seasons, $P$. amboinicus yielded $0.10 \%$ oil and had thymol as major component. M. pulegium yielded $0.20 \%$ and $0.09 \%$ oil in the spring and winter, respectively. The major components were pulegone and trans-caryophyllene, whereas borneol, menthol and piperitone were identified at small quantities. Higher menthol and isomenthol levels were detected in the winter. In addition, neoisomenthyl, menthyl and isomenthyl acetates were only observed in the winter. This work contributes to the knowledge of plant species grown in southern Bahia.
\end{abstract}

Key words: Lamiaceae, Ilhéus, Bahia, spring, winter

\section{INTRODUÇÃO}

A grande extensão territorial do Brasil, as diversas situações climáticas, geomorfológicas e de solo resultam na mais diversificada flora do mundo, com cerca de 60.000 espécies de vegetais superiores (Barreiro \& Bolzani, 2009). As plantas medicinais integram essa biodiversidade. No Nordeste do Brasil, grande parte dos habitantes da região usa o conhecimento de gerações para tratar de enfermidades e procurar manter-se sadios (Silva et al., 2000). Calcula-se que $80 \%$ da população dos países subdesenvolvidos e em desenvolvimento são quase completamente dependentes da medicina caseira, utilizando plantas para as necessidades primárias de saúde (Braz-Filho, 1994). O uso de plantas medicinais é crescente, vários produtos vêm sendo utilizados com diferentes aplicações, no consumo de medicamentos fitoterápicos e complementos alimentares. Isso se deve a algumas variáveis sociais tais como hábitos, concepções, conhecimentos, tradições, opiniões, valores e costumes. A ciência busca unificar o progresso com aquilo que a natureza oferece para tal, deve-se

Recebido para publicação em 05/05/2009

Aceito para publicação em 03/09/2010

Rev. Bras. PI. Med., Botucatu, v.13, n.2, p.165-169, 2011. 
respeitar a cultura do povo em torno do uso de produtos ou ervas medicinais para curar os males (Accorsi, 2000).

A produção dos metabólitos secundários nas plantas é susceptível as fatores genéticos, ambientais e morfológicos (Simões et al., 2000) sendo imprescindível a caracterização química das plantas cultivadas nas diversas regiões do nosso País. O estudo químico, além de contribuir para o conhecimento da flora local, propicia o desenvolvimento de novos fármacos e contribui para assegurar a população 0 uso adequado e seguro das tradições populares.

A comunidade da Vila Cachoeira, em Ilhéus (Bahia) faz uso de plantas medicinais da família Lamiaceae (Moreira et al., 2002). Poejo, Mentha pulegium L. e o hortelã-grosso, Plectranthus amboinicus (Lour.) Spreng., são exemplos. $M$. pulegium, conhecida popularmente como poejinho, poejo-real e menta-selvagem é usada pela comunidade no combate a febre (Moreira et al., 2002). Atua como digestivo, expectorante e antiespasmódico (Lorenzi et al., 2002). Em uso tópico é usada como cicatrizante e antisséptico.

São conhecidos três quimiótipos de $M$. pulegium: 1) pulegona, 2) piperitenona e ou piperitona e 3) isomentona e neoisomentol (Cook et al., 2007). $\mathrm{Na}$ Bulgária, o óleo essencial apresenta $40 \%$ de pulegona, $20 \%$ de piperitenona e $2 \%$ de isomentona (Stoyanova et al., 2005). O óleo essencial extraído do cultivar no Himalaia apresenta $75 \%$ de pulegona, $8 \%$ de mentona, $3 \%$ de isomentona, $1 \%$ de neomentol e $2 \%$ de piperitona (Agnihotri et al., 2005). Na Grécia, óleos essências extraídos da inflorescência, folhas e pedúnculos apresentaram diferentes quantidades de pulegona, variando de $32,8-75,8 \%$ (Cook et al., 2007). Na Austrália, essa espécie é rica em piperitona $(70 \%)$, apresenta também limoneno (11\%), mentona ( $8 \%$ ) e isomentona (7\%) (Zwaving et al., 1971). Pavela (2005), na República Tcheca, observou ação tóxica do óleo essencial na larva de Spodoptera littoralis.

A presença de pulegona, substância tóxica (Martins et al., 2000) no óleo essencial da M. pulegium incentiva estudos do uso como repelente e mostra a importância de difundir o conhecimento químico dos metabolitos secundários desta planta visando o uso seguro da mesma.

O hortelã-grosso, Plectranthus amboinicus (Lour.) Spreng., é popularmente conhecido como hortelã-grande, hortelã-graúda e hortelã-gorda. Na Vila Cachoeira é utilizada no tratamento tosse, gripe, verme e hemorróidas (Moreira et al., 2002). Para essa espécie são relatados dois quimiótipos, um rico em timol e outro em carvacrol (Murthy \& Srinvas, 2009). $\mathrm{Na}$ Índia, o óleo essencial de $P$. amboinicus é rico em carvacrol (70\%) e apresenta propriedades fungitóxicas frente à Aspergillus flavus, Aspergillus niger, Candida versatilis e Fusarium $s p$ (Murthy \& Srinvas, 2009). No Ceara, o óleo essencial dessa espécie também é rico em carvacrol $(68 \%)$ e mostra toxicidade frente ao microcustáceo Artemia salina (Pereira et al., 2008). Apesar da escassez de estudos sobre sua eficácia e segurança, $P$. amboinicus é amplamente usada no Brasil (Lorenzi et al., 2002; Costa, 2006).

Nesse trabalho, foi analisada a composição química dos óleos essenciais de duas plantas medicinais usadas na comunidade de llhéus, $M$. pulegium e $P$. amboinicus, cultivados na primavera e no inverno.

\section{MATERIAL E MÉTODO}

Coleta de Material: As espécies foram coletadas na Vila Cachoeira, localizada na Rodovia Ilhéus-Itabuna, Km 13, longitude 39008'96"W e latitude 14²8'55"S, município de llhéus em 2007, nos meses de agosto (inverno) e novembro (primavera). As plantas, coletadas no período da manhã, foram acondicionadas e transportadas para o Laboratório de Pesquisa em Produtos Naturais e Síntese Orgânica (LPPNS), na Universidades Estadual de Santa Cruz (UESC). Exsicatas das espécies encontram-se depositadas no Herbário da Universidade, sob os números 3168 (M. pulegium) e 2519 ( $P$. amboinicus), com as determinações feitas pela professora Dra Larissa Corrêa do Bonfim Costa, da UESC.

Extração dos óleos: As folhas frescas foram submetidas ao processo de hidrodestilação usando adaptador Clevenger por duas horas. Após a hidrodestilação foram realizadas extrações por partição líquido-líquido, utilizando como solvente orgânico o diclorometano para a $P$. amboinicus e éter etílico para a M. pulegium. A fase orgânica foi secada com sulfato de sódio anidro e concentrada. As extrações foram realizadas em triplicatas. O teor de óleo essencial foi determinado pelo volume dos óleos extraídos e expresso em percentagem $\mathrm{m} / \mathrm{v}$ ( $\mathrm{mL}$ de óleo por $100 \mathrm{~g}$ de matéria fresca).

Analise dos óleos essenciais: As amostras de óleos foram analisadas por cromatografia gasosa, utilizando o aparelho Varian Saturno 3800, localizado no laboratório de Fisiologia Vegetal da UESC, equipado com detector de ionização de chama (FID), utilizando coluna capilar de sílica fundida VA-1 (30 m x $0,25 \mathrm{~mm} \times 0,25 \mu \mathrm{m}$ ) tendo hélio como gás arraste, fluxo de $1,4 \mathrm{~mL} \mathrm{~min}^{-1}$. As temperaturas do injetor e detector foram de $250^{\circ} \mathrm{C}$ e $280^{\circ} \mathrm{C}$, respectivamente. A temperatura da coluna para as análises foram programadas tendo início a $60^{\circ} \mathrm{C}$, aumentando $8^{\circ} \mathrm{C}$ até $260^{\circ} \mathrm{C}$. Para as análises dos óleos da espécie $M$. pulegium foram injetados $1 \mu \mathrm{L}$ de solução a $5 \%$ de

Rev. Bras. PI. Med., Botucatu, v.13, n.2, p.165-169, 2011. 
óleo em clorofórmio, com as seguintes razões split 1:10 até o tempo de retenção de 12,5 minutos mudando para $1: 100$ de 12,5 minutos até 15,2 minutos e retornando ao splitde 1:10. Para as análises dos óleos de $P$. amboinicus foram injetados $1 \mu \mathrm{L}$ de solução em clorofórmio a 10\% nas seguintes razões split 1:100 até tempo de retenção 13:00 minutos mudando para 1:50 a partir do tempo de retenção 15:00. A concentração dos constituintes voláteis foi calculada através da área da integral de seus respectivos picos, relacionadas com a área total de todos os constituintes da amostra. As análises qualitativas dos óleos foram realizadas usando-se o cromatógrafo a gás HP6890 acoplado ao espectrômetro de massas HP5989A. Foi usada coluna PDMS ( $50 \mathrm{~m} \times 0,20 \mathrm{~mm}$ com $0,2 \mu \mathrm{m}$ de espessura de filme) tendo hélio como gás de arraste $\left(1,5 \mathrm{~mL} \mathrm{~min}^{-1}\right)$, temperatura do detector e injetor de $300^{\circ} \mathrm{C}$. Foram injetados $1 \mu \mathrm{L}$ no modo split (1:22). $\mathrm{O}$ modo de operação foi impacto eletrônico a $70 \mathrm{eV}$. As programações de temperatura da coluna foram às mesmas usadas nas análises de cromatografia a gás. Os diversos constituintes químicos dos óleos essenciais foram identificados através da comparação computadorizada com a biblioteca do aparelho, literatura e índice de retenção de Kovats (Adams, 1995). Os índices de retenção de Kovats (IK) foram calculados através da injeção de uma série de padrões de $n$-alcanos $\left(\mathrm{C}_{8}-\mathrm{C}_{26}\right)$, injetados nas mesmas condições cromatográficas das amostras.

\section{RESULTADO E DISCUSSÃO}

Os teores dos óleos essenciais de $P$. amboinicus extraídos, na primavera e inverno, foram idênticos, ao contrário da espécie $M$. pulegium que forneceu na primavera $120 \%$ a mais de óleo comparado ao inverno (Tabela 1).

As análises cromatográficas dos óleos de

TABELA 1. Principais componentes voláteis de M. pulegium e $P$. amboinicus.

\begin{tabular}{|c|c|c|c|c|c|}
\hline \multicolumn{2}{|l|}{ Espécie } & \multicolumn{2}{|c|}{ M. pulegium } & \multicolumn{2}{|c|}{ P. amboinicus } \\
\hline & & Primavera & Inverno & Primavera & Inverno \\
\hline Teor (\%) & & 0,20 & 0,09 & 0,10 & 0,10 \\
\hline Componentes & $\mathbb{K}^{*}$ & & & & \\
\hline orto-cimeno & 1026 & & & 0,53 & \\
\hline 1,8-cineol & 1027 & 0,29 & 1,53 & & \\
\hline para-cimeno & 1036 & & & 0,16 & \\
\hline (Z)- $\beta$-ocimeno & 1041 & & 1,04 & & \\
\hline$\gamma$-terpineno & 1059 & & & 0,69 & \\
\hline borneol & 1165 & 6,62 & 8,87 & & \\
\hline mentol & 1175 & 6,75 & 9,97 & & \\
\hline isomentol & 1182 & 0,73 & 2,99 & & \\
\hline terpine-4-ol & 1186 & & & 1,23 & 0,89 \\
\hline neoisomentol & 1197 & & 0,62 & & \\
\hline pulegona & 1258 & 61,43 & 28,4 & & \\
\hline piperitona & 1267 & 2,29 & 6,13 & & \\
\hline neoisomentila & 1280 & & 2,18 & & \\
\hline acetato de mentila & 1296 & & 2,50 & & \\
\hline acetato de isomentila & 1313 & & 1,64 & & \\
\hline trans-cariofileno & 1397 & 18,68 & 10,20 & & \\
\hline timol & 1421 & & & 75,39 & 70,28 \\
\hline E-cariofileno & 1435 & & & 10,52 & \\
\hline a-humuleno & 1468 & & & 2,26 & 1,75 \\
\hline$\beta$-bisaboleno & 1501 & & & 0,39 & \\
\hline oxido de cariofileno & 1605 & & & & 2,62 \\
\hline
\end{tabular}

*Indice Kovats experimental determinado em coluna VA-1 
P. amboinicus permitiram a identificação de $91,17 \%$ dos componentes na primavera e $75,54 \%$ no inverno (Tabela 1). O componente majoritário, nas duas estações, foi o arilpropanóide (Ar) timol, 75,39\% na primavera e $70,28 \%$ no inverno. Os óleos apresentam também monoterpenos $(\mathrm{M})$, monoterpenos oxigenados (MO) e sesquiterpenos (S) (Figura 1). E-cariofileno $(10,52 \%)$ foi identificado somente na primavera enquanto o derivado oxigenado, óxido de cariofileno $(2,62 \%)$, somente no inverno. O $\alpha$-humuleno e terpine4-ol foram observados em maior quantidade na primavera, 2,26\% e 1,23\%, respectivamente. O óleo essencial de $P$. amboinicus cultivado em llhéus pertence ao quimiótipo timol.
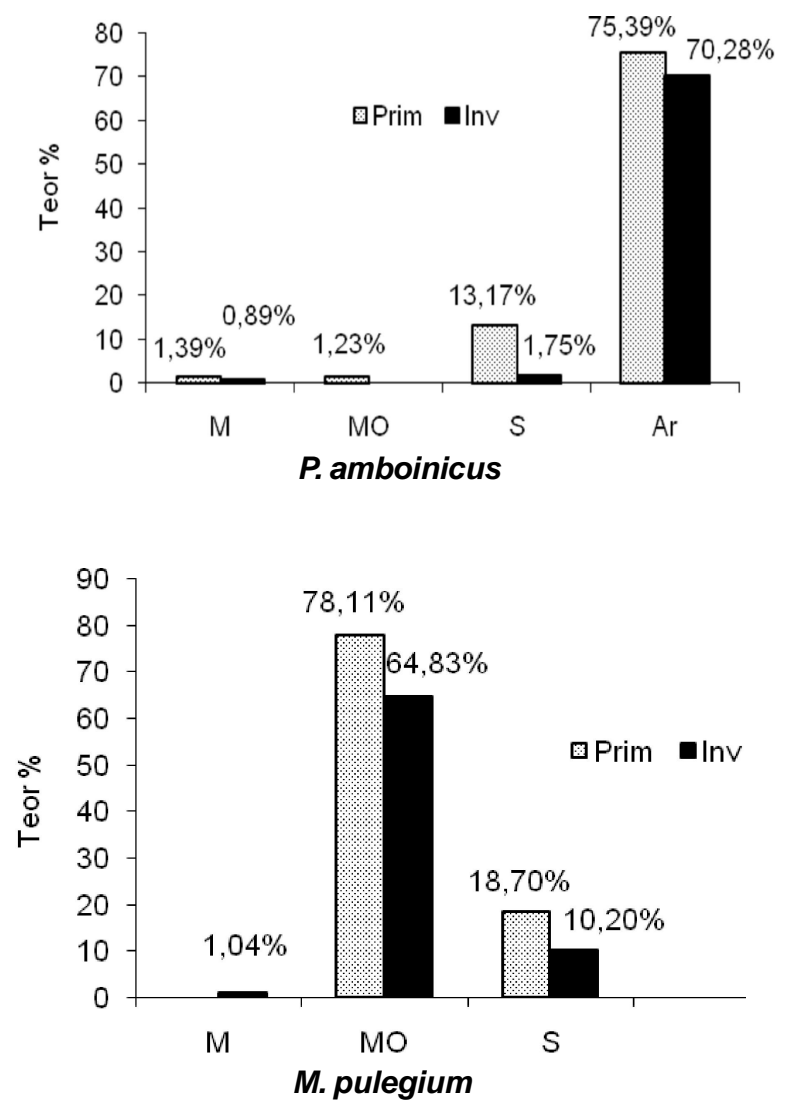

FIGURA 1. Classe de compostos voláteis dos óleos essenciais.

As análises cromatográficas dos óleos essenciais da M. pulegium permitiram a identificação de $96,79 \%$ de componentes voláteis na primavera e $76,07 \%$ no inverno. Os óleos são ricos em monoterpenos oxigenados (Figura 1). Apresentam em menores quantidades compostos sesquiterpênicos. Na primavera foram detectados 6,62\% de borneol e $61,43 \%$ de pulegona e no inverno, $8,87 \%$ de borneol e $28,40 \%$ de pulegona. Houve redução de aproximadamente $54 \%$ na produção da pulegona no inverno, por outro lado, foram identificados derivados de mentona, diferentemente do óleo extraído na primavera. Derivados do mentol e piperitona apresentam teores maiores no inverno. De acordo com a proposta de Croteau (2000), a pulegona é a substância precursora para a formação de estereoisômeros da mentona, os quais originam derivados acetilados de mentila, de neoisomentila e de isomentila, diferentemente do óleo extraído da primavera, no qual não foi observada a formação dessas substâncias. As condições extrínsecas de cultivo na primavera propiciam a formação da maior quantidade de pulegona e não a conversão em estereoisômeros da mentona. Os óleos extraídos, tanto na primavera como no inverno, pertencem ao mesmo quimíotipo da espécie M. pulegium, rico em pulegona.

A presença de borneol e pulegona, substâncias tóxicas, em plantas medicinais restringe o uso das mesmas (Lorenzi et al., 2002). Portanto, corroborando com Martins e colaboradores (2000), a presença de borneol na espécie M. pulegium restringe o uso por grávidas, principalmente nos três primeiros meses. A presença da pulegona nesses óleos é indicativa de atividades macrobióticas, inseticidas e repelentes (Lorenzi et al., 2002).

O óleo essencial de $P$. amboinicus, cultivado na Vila Cachoeira, difere do quimiótipo cultivado no Ceará, estado também pertencente à região do nordeste do Brasil. A presença de timol é um indicativo do potencial uso como bactericida.

Esse trabalho contribui para o conhecimento das plantas medicinais usadas pela comunidade da Vila Cachoeira, no Sul da Bahia.

\section{AGRADECIMENTO}

A UESC pelo suporte financeiro, a Fundação de Amparo a Pesquisa de Estado da Bahia (FAPESB) pela concessão da bolsa de iniciação cientifica e ao Sr. Arnaldo Silva pelo cultivo das espécies.

\section{REFERÊNCIA}

ACCORSI, W.R. Medicina natural, um novo conceito. A fórmula: guia de negócios. Revista Espaço para a Saúde, v.2, n.4, p.5-8, 2000.

ADAMS, R.P. Identification of essential oils by gas chromatography/mass spectroscopy. Allured: Publ. Corp. Carol Stream IL, 1995. 468p.

AGNIHOTRI, V.K. et al. Essential oil composition of Mentha pulegium L. growing wild in the north-western Himalayas India. Flavour and Fragrance Journal, v.20, n.6, p.607-10, 2005.

BARREIRO, E.J.; BOLZANO,V.S. Biodiversidade:fonte potencial para a descoberta de fármacos. Química Nova, v.32, n.3, p.679-88, 2009.

BRAZ-FILHO, R. Química de produtos naturais: importância, interdisciplinaridade, dificuldades e perspectivas. Química Nova, v.17, n.5, p.405-9, 1994.

COOK, C.M. et al. Differences between the inflorescence,

Rev. Bras. PI. Med., Botucatu, v.13, n.2, p.165-169, 2011. 
leaf and stem essential oil of wind Mentha pulegium plants from Zakynthos, Greece. Journal of Essential Oil Research, v.19, p.239-43, 2007.

COSTA, M.C.C.D. Uso popular e ações farmacológicas de Plectranthus barbatus Andr. (Lamiaceae): revisão dos trabalhos publicados de 1970 a 2003. Revista Brasileira de Plantas Medicinais, v.8, n.2, p.81-8, 2006.

CROTEAU, R.; HUTCHAN, T.M.; LEWIS, N.G. Natural products (secondary metabolites). In: BUCHANAM, B.B. et al. Biochemistry \& molecular biology of plants. Rockville: Courier Companies, 2000. p.1250-318.

LORENZI, H. et. al. Plantas medicinais no Brasil: nativas e exóticas. Nova Odessa: Instituto Plantarum, 2002. 544p. MARTINS, E. et al. Plantas medicinais. Viçosa: UFV, 2000. 220p.

MOREIRA, R.C.T. et al. Abordagem etnobotânica acerca do uso de plantas medicinais na Vila Cachoeira, Ilhéus, Bahia, Brasil. Acta Farmacêutica Bonaerense, v.21, n.3, p.1-7, 2002.

MURTHY, P.S.; SRINVAS, R.P. Fungitoxic activity of Indian borage (Plectranthus amboinicus) volateis. Food Chemistry, v.14, p.1014-8, 2009.
PAVELA, R. Insecticidal activity of some essential oils against of Spodoptera littoralis. Fitoterapia, v.76, n.7-8, p.691-6, 2005.

PEREIRA, C.K.B. et al. Composição química e toxicidade do óleo essencial de Plectranthus amboinicus (Lour) Spreng. In: REUNIÃO REGIONAL FeSBE, 3., 2008, Fortaleza-Ceará. Resumos... Ceará, 2008. Disponível em: <http://www.fesbe.org.br/regional2008/?resumos/ 36.003 >. Acesso em: 30 jan. 2009.

SIMÕES, C.M.O. et al. Farmacognosia: da planta ao medicamento. 2.ed. Porto Alegre/Florianopólis: Editora UFRGS/Editora UFSC, 2000. 821p.

SILVA, P.B. et al. O papel do professor na produção de medicamentos fitoterápicos. Química Nova na Escola, v.11, p.19-21, 2000.

STOYANOVA, A. et al. Chemical composition of the essential oil of Mentha pulegium L. from Bulgaria. Journal of Essential Oil Research, 2005. Disponivel em: $<$ http://www.Scielo.br>. Acesso em: 10 jan. 2008.

ZWAVING, J.H.; SMITH, D. Composition of the essential oil of Austrian Mentha pulegium. Phytochemistry, v.10, n.8, p.1951-3, 1971. 